\title{
Dichotic, diotic, and monaural summation of loudness: A comprehensive analysis of composition and psychophysical functions
}

\author{
DANIEL ALGOM \\ John B. Pierce Foundation Laboratory, New Haven, Connecticut \\ Yale University, New Haven, Connecticut \\ and Bar Ilan University, Ramat Gan, Israel \\ and \\ BILHA BEN-AHARON and LIOR COHEN-RAZ \\ Bar Ilan University, Ramat Gan, Israel
}

\begin{abstract}
In a series of six experiments, the method of magnitude estimation, constrained by a multivariate model, was used to assess the rules that govern the summation of the loudness of two-tone complexes. This methodology enabled us to specify the amounts of summation and simultaneously to construct the corresponding loudness scales. The components had different frequency separations and in the different experiments were presented (1) dichotically, a different frequency to each ear; (2) diotically, to both ears; and (3) monaurally. Results replicated and in some conditions extended known features of multiple signal processing by the auditory system. Thus, qualitatively different rules of loudness integration appeared. For monaural and diotic modes of stimulation, overall loudness depended on total sound energy within the critical band, but on the simple sum of component loudnesses beyond the critical band. For dichotic presentations, a fully additive rule of loudness summation appeared, regardless of frequency spacing. For the latter (but not the former), loudness summation was perfect, with the underlying loudness scales closely approximating Stevens's sone scale.
\end{abstract}

In the present set of experiments, we measured the loudness of two tones as a function of (1) their frequency separation (narrow spacing within a critical band vs. wide spacing across a critical band) and (2) their mode of presentation (dichotic, diotic, or monaural). Although the various conditions thus generated have all come under the name of auditory summation, there are substantial differences among them in both the nature of the subjective experience and the objective, measurable outcome. For one, they can be classified as analytic or synthetic, depending on whether or not the components of stimulus mixtures are perceptually separable. More importantly, the rules of integration may differ across conditions. Indeed, the major purpose of this study is to uncover systematically the rules that govern summation under specific conditions of stimulation. Both of the variables tested within the framework of the present investigation are shown to affect the way the auditory system deals with the effects of concurrent multiple stimulation.

Preparation of this paper was supported by NIH Grant NS21326 to Lawrence Marks. His support and suggestions are gratefully acknowledged, as is Robert Melara's useful criticism. We also thank Edward Carterette and two anonymous reviewers for helpful comments on an earlier version of this manuscript. Barbara Faulkner and Elise Low provided skillful assistance at various stages of the completion of the manuscript. Address correspondence to Daniel Algom, Department of Psychology, Bar Ilan University, Ramat Gan 52100, Israel.
Many of the basic findings on loudness summation have been reported by earlier researchers (e.g., Algom \& Marks, 1984; Irwin, 1965; Marks, 1978a, 1978b, 1979a, 1979b, 1980; Porsolt \& Irwin, 1967; Reynolds \& Stevens, 1960; Scharf, 1969, 1970, 1978; Scharf \& Fishken, 1970; Zwicker, Flottorp, \& Stevens, 1957; Zwicker \& Scharf, 1965). In the great bulk of this research, matching or various versions of adjustment have been used as the main means for gauging summation. However, measured differences (say, in decibels) between monaural and binaural (or different frequency) sounds that subjectively match for loudness do not uniquely determine the amount of summation in the sense that they do not specify loudness units. The degree of loudness summation depends on (1) the form of the psychophysical function for loudness (particularly, whether or not it is a power function); (2) the parameters of the loudness function (e.g., the size of the exponent in the case of a power relation); and (3) whether (1) and (2) are the same for monaural and binaural loudness, or for loudness of sounds of different frequencies. Also, in earlier studies, simple binaural or spectral stimuli were mainly tested (the stimuli consisted of equal intensity levels to the two ears or at different frequencies).

By contrast, in the present study, we used judgments of loudness based on a factorial presentation of the component variables, yielding tightly constrained metric struc- 
tures. Given the appropriate constraints (e.g., linear additivity), these allow for unique and independent derivation of both the psychophysical functions and the associated rules of loudness combination. When the stimulus array comprises various combinations of equal and unequal sound pressure levels (SPLs), the rank-order relations among the loudness judgments alone suffice to uncover the underlying scale of loudness and the rules of concatenation (see Marks, 1987). Hence, influence on the results of various response biases (e.g., Algom \& Marks, 1984; Schneider, 1980) can be avoided altogether. A stimulus set of this type, therefore, makes a sharper delineation of the different integration rules and scales of loudness that have been suggested as governing the various loudness summation phenomena.

\section{Diotic, Dichotic, Subcritical, and Supercritical Loudness Summation Reconsidered}

The basic features characterizing binaural, dichotic, subcritical, and supercritical loudness integration have been documented (e.g., by Algom, Adam, \& Cohen-Raz, 1988; Algom \& Marks, 1984; Marks, 1978a, 1978b, 1979a, 1979b, 1980; Scharf, 1970, 1978). Loudness shows (1) a superiority of binaural to monaural listening, (2) invariance within a critical band, but (3) dependence on frequency separation beyond the critical band (diotic stimulation). For supercritical sounds, loudness increases with overall frequency separation, regardless of whether the components are fed to a single ear or binaurally to both. However, when signals are presented dichotically, with a different frequency component at each ear, loudness does not depend on the frequency separation between the left-ear and right-ear components (Niese, 1960; Porsolt \& Irwin, 1967; Scharf, 1969). The critical bandwidth limitation only affects monarual (or diotic binaural) inputs, and it does not apply to dichotic modes of stimulation. ${ }^{1}$

\section{The Present Research}

In the present study, we aim to take another look at the way(s) in which the auditory system integrates sensory inputs across frequency and across the two ears. We probe four related loudness-summation phenomena-namely, critical-band, supercritical-band, diotic, and dichotic summation-within the framework of a single comprehensive investigation. Although attributes of these processes have been studied in the past, separately and in several subcombinations, all have not been subjected to an encompassing, systematic examination, according to common methods of experimentation and analysis.

Indeed a significant feature of the present experiments is the composition of stimuli. We used a matrix of stimuli in which each of several SPLs at a given frequency was combined with each of several SPLs at another frequency. The resulting two-tone complexes were presented monaurally, diotically to both ears, or dichotically (one frequency to each ear) in the different experiments. These three experimental conditions were replicated twice for
Table 1

Overview of the Research Program via a Two-Way Classification of Experiments

\begin{tabular}{ccc} 
Mode of & \multicolumn{2}{c}{$\begin{array}{c}\text { Frequency Separation } \\
\text { of Components }\end{array}$} \\
\cline { 2 - 3 } $\begin{array}{c}\text { Within a } \\
\text { Presentation }\end{array}$ & $\begin{array}{c}\text { Beyond a } \\
\text { Critical Band }\end{array}$ & Crical Band \\
\hline Dichotic & Experiment 1 & Experiment 4 \\
Diotic & Experiment 2 & Experiment 5 \\
Monaural & Experiment 3 & Experiment 6 \\
\hline
\end{tabular}

different frequency spacings of the two-tone complexes. The latter comprised either narrowly spaced, subcritical components or widely spaced supercritical components. The resulting six experiments are summarized in Table 1. To anticipate-whereas the data in some of the specific conditions are a bit noisy, the results, taken as a whole, disclose general properties of the auditory system's behavior in the summation domain.

\section{EXPERIMENT 1: DICHOTIC SUMMATION OF THE LOUDNESS OF TONES OF NARROW FREQUENCY SEPARATION}

In this experiment, pure tones of 1000 and $1100 \mathrm{~Hz}$ were presented dichotically, a different frequency to each ear. Note that the spacing of the two tones is well within the width of the critical band (whose value is about $170 \mathrm{~Hz}$ around a center frequency equal to their geometric mean). Yet one of the tones is presented to the left ear and the other to the right ear. Thus we may bypass the peripherally based critical-band mechanism, resulting in (perfect) summation that is usually demonstrated for equalfrequency binaural signals (Algom \& Marks, 1984; Marks, 1978a, 1979b). On the other hand, the present dichotic summation should differ from monaural or diotic summation, for the latter inputs are affected by the critical-band mechanism. Indeed Scharf (1969) as well as Porsolt and Irwin (1967) reported data compatible with this prediction. However, a factorial design of the type used in the present experiment yields additional constraints on the resulting loudness properties, thereby providing a more rigorous test of the rule of summation.

\section{Method}

Sinusoidal signals from two oscillators (Heathkit Audio Generator, Model IG-72) provided pure-tone 1000- and 1100-Hz signals. Each sinusoid could be amplified and attenuated independently. They were gated and timed (with locally designed crystal-controlled timers sustained by appropriate logic and circuitry) to produce 1$\mathrm{sec}$ stimuli with rise and decay times of $1 \mathrm{msec}$. These signals were led into a sound-attenuated chamber (Medtechnic Silent Cabin), where they fed AKG (K150) Headphones placed in 250-A cushions. The stimuli were calibrated with a Brüel and Kjaer Type 2204 Impulse Precision Sound Level Meter. All durations and frequencies were monitored with a Monsanto Type 120A Counter-Timer.

Six different levels of sound pressure $(30,40,45,50$, and $55 \mathrm{~dB}$ SPL, as well as a level well below threshold) at $1000 \mathrm{~Hz}$ to one ear were combined with the same six levels at $1100 \mathrm{~Hz}$ to the other ear, making 36 different stimuli in all. For a random half of the subjects, the $1000-\mathrm{Hz}$ component was presented to the left ear and 
the $1100-\mathrm{Hz}$ component to the right ear, whereas for the others, the 1100-Hz component appeared at the left ear and the $1000-\mathrm{Hz}$ tone at the right ear. Each stimulus complex was presented and judged four times in the course of an experimental session. The order of presentation of stimuli was irregular (orderly sequences that occasionally arise in random selection were intentionally avoided) and different for each subject.

The method consisted of magnitude estimation. The subjects were instructed to assign to the first stimulus whatever number seemed most appropriate to represent its loudness; then, the subjects were instructed to assign numbers, in proportion, to the succeeding stimuli. If no sound was heard, the subjects were to assign the number zero. The subjects were told that they could use whole numbers, decimals, and fractions, as needed. The subjects were instructed explicitly to judge the overall loudness of the dichotic complex (rather than any one of the monaural components).

Ten volunteers from the Bar-Ilan community ( 7 males and $3 \mathrm{fe}$ males, between 22 and 35 years of age) served as subjects. Three had had previous experience with the method of magnitude estimation, although not necessarily in judging loudness.

\section{Results and Discussion}

The magnitude estimates given for each stimulus were brought to a common modulus (Lane, Catania, \& Stevens, 1961) for all subjects, and then they were averaged geometrically. These means are plotted in Figure 1 as a function of the SPL of the $1000-\mathrm{Hz}$ signal delivered to either

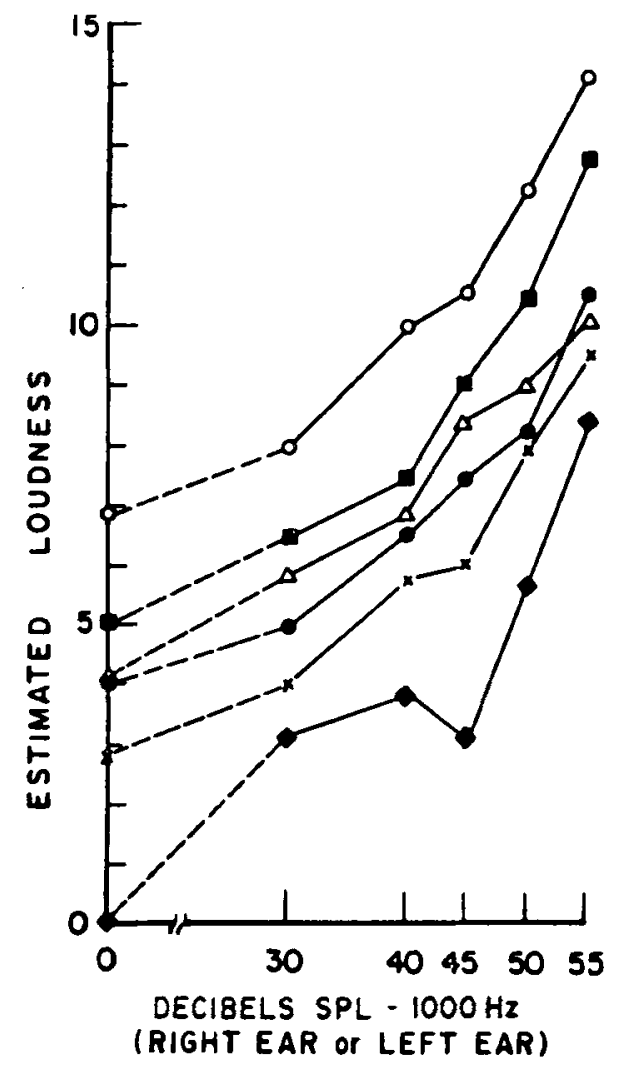

Figure 1. Dichotic summation of loudness. Magnitude estimates of the loudness of a two-tone complex $(1000-1100 \mathrm{~Hz})$, as a function of the SPL at $1000 \mathrm{~Hz}$, delivered to a single ear. Each contour represents one of the same levels at $1100 \mathrm{~Hz}$, delivered to the other ear. (From bottom in ascending order: 0, 30, 40, 45, 50, and $55 \mathrm{~dB}$ SPL.)

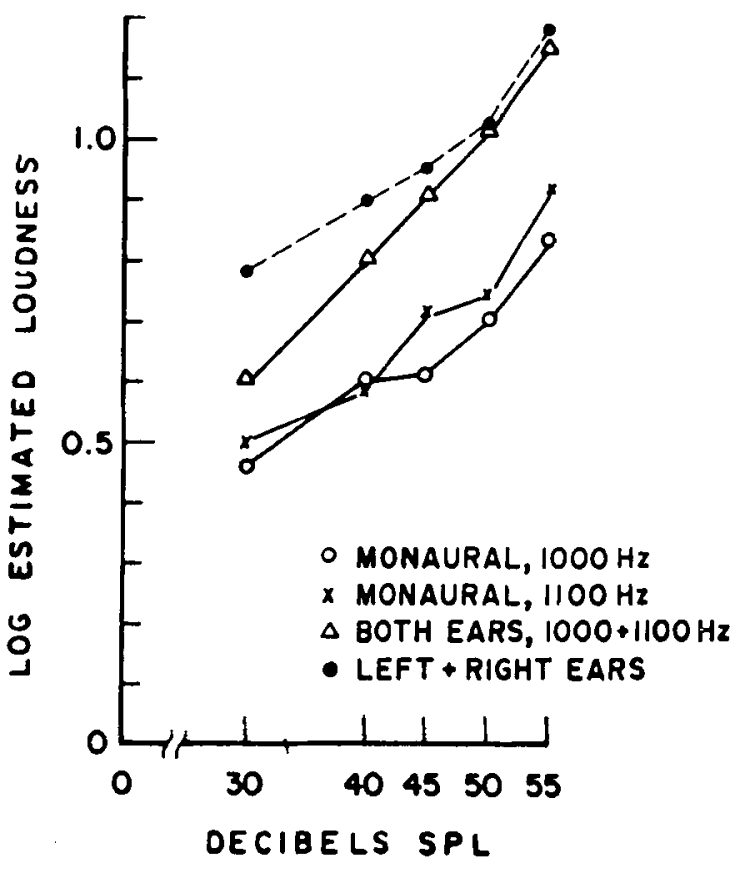

Figure 2. Single-component monaural (1000 and $1100 \mathrm{~Hz})$, and dichotic $(1000-1100 \mathrm{~Hz})$, loudness functions.

the right or the left ear. Each contour represents a different constant SPL at $1100 \mathrm{~Hz}$ delivered to the other ear. Note that the loudness estimates are plotted on a linear scale; this makes it possible to assess by means of visual inspection the rule of loudness summation.

A salient characteristic of this family of curves is that, to a good first approximation, the different functions have about the same shape, but they are displaced from one another in the vertical dimension. Observed parallelism, in turn, implies a rule of linear additivity (Algom \& Marks, 1984; Anderson, 1970, 1982; Marks, 1978a). The magnitude estimates assigned to a given stimulus approximate the sum of the estimates given to the left-ear and right-ear components individually.

The model of linear additivity can be given a statistical test. Often it is possible to accomplish this by applying analysis of variance to the data (Anderson, 1970). Legitimate application of analysis of variance, however, requires that the data have moderately uniform variance, a condition that seldom holds with magnitude estimates (cf. Algom \& Cohen-Raz, 1984; Marks, 1980). Nevertheless, the present data had sufficiently uniform variances to justify application of analysis of variance in this case. The interaction is the critical term to assess, because failure of additivity will appear as a significant interaction (Anderson, 1970). The data showed a nonsignificant overall interaction $[F(25,125)=1.03, p>.05]$, confirming statistically the results of the graphical test "of parallelism: linear addition of the loudnesses of differentfrequency tonal signals across the two ears.

In Figure 2, a subset of these data is plotted-namely, the results obtained when the $1000-$ and $1100-\mathrm{Hz}$ com- 
ponents were presented singly (hence monaurally), and those obtained for simple dichotic presentations (equal SPLs to the two ears). The dashed line (filled circles) shows the sum of the magnitude estimates of the 1000(circles) and $1100-\mathrm{Hz}(\times$ s) monaural stimuli. The dashed line passes close to the actual magnitude estimates given to the dichotic stimuli (triangles) at high values, but a large departure is apparent at the low end. The data, then, come close to agreeing with a simple additive model, but fall short of additivity at low SPLs.

What loudness scale underlies the dichotic summation of narrowly spaced pure tones? As noted in the introduction, this is a crucial question to answer if one is to specify the degree of summation. Both the $1000-$ and $1100-\mathrm{Hz}$ monaural functions and the dichotic function approximate power functions (straight lines in the log-log coordinates). The (monaural) power functions for pure tones of 1000 and $1100 \mathrm{~Hz}$ have exponents of $0.32\left(r^{2}=.935\right)$ and 0.28 $\left(r^{2}=.945\right)$, respectively. The dichotic loudness function has a greater exponent of 0.42 , with a better fit $\left(r^{2}=\right.$ .998). These exponents are notably smaller than 0.6 , the value that Stevens gave in 1956 to govern the sone scale. The small exponents presumably reflect the complexity of the stimulus array. (Note in this respect that the 1000and $1100-\mathrm{Hz}$ functions, though nominally monaural, are not, in fact, derived from the same ear in all subjects.) Interestingly, however, most summation studies report remarkably low power function exponents even for simple pure-tone stimuli (i.e., equal SPLs to the two ears; see Marks, 1979a, 1980, 1987; Scharf, 1969; Scharf \& Fishken, 1970).

Another indicant of the underlying scale values comes from taking marginal means (generated by summing down columns and across rows of the response matrix and then setting to zero the scale values at zero stimulus intensity). Because they are derived from the entire response matrix, the marginal means have a fuller basis than do the monaural judgments. The marginal means for the 1000 and $1100-\mathrm{Hz}$ tones are plotted in Figure 3.

The single line drawn in Figure 3 provides a reasonably good description of the way loudness grows for equallevel, hence almost equally loud, $1000-$ and $1100-\mathrm{Hz}$ tonal stimuli. Although there is a small difference in exponent (b) between the power functions for the two frequencies (for $1000 \mathrm{~Hz}, b=0.43, r^{2}=.978$; for $1100 \mathrm{~Hz}, b=$ $0.52, r^{2}=.999$ ), a single loudness function with an exponent of 0.46 provides good fit $\left(r^{2}=.994\right)$ to both data sets. In fact, the parallel monaural functions in Figure 2 reveal similarly minor differences in loudness between the two frequencies.

Perfect dichotic summation means that the loudness of a dichotic tone pair equals the sum of the input loudnesses of the two components (cf. Scharf, 1969). Since in the present experiments the 1000 - and $1100-\mathrm{Hz}$ components were about equally loud (for equal SPL), overall loudness should be twice that of either component. The obtained average dichotic ratio for equal SPL tones was $1.72: 1$. This ratio reflects the fact that although both the

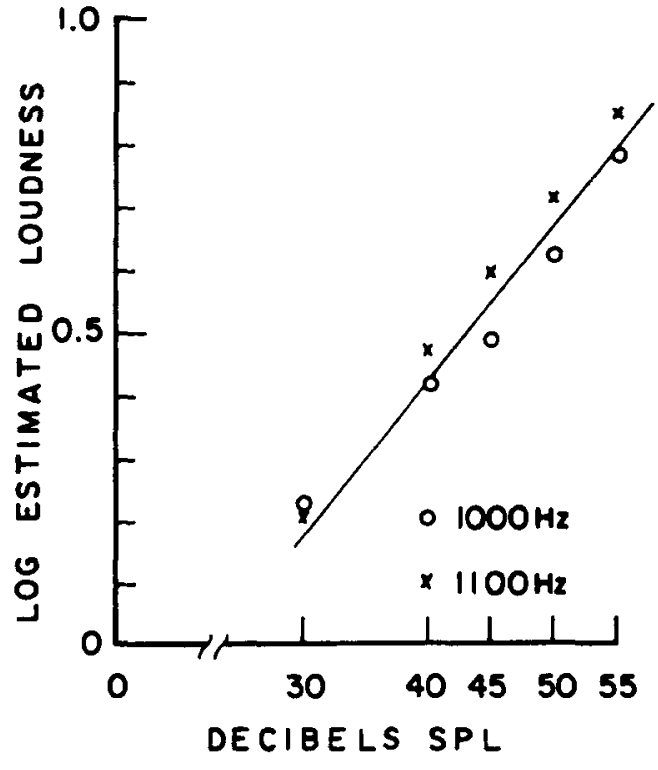

Figure 3. Average loudness function at 1000 and $1100 \mathrm{~Hz}$, derived from the marginal means of the data plotted in Figure 1.

1000 - and the $1100-\mathrm{Hz}$ functions were separated horizontally from the dichotic function by about $10 \mathrm{~dB}$ (Figure 2), all are governed by a power function exponent of approximately 0.46 . If, however, the numerical estimates are rescaled by means of a mild monotonic power transform to bring them to agree with sones (this entailed raising the magnitude estimates to the $0.6 / 0.46=1.3$ power), dichotic summation becomes 2.02:1. Thus, there is an intrinsic consistency between measurement of loudness in sones and complete dichotic summation. A 10-dB horizontal separation between the dichotic and single-frequency loudness functions means complete summation in sones, because a $10-\mathrm{dB}$ dichotic gain corresponds to a $2: 1$ ratio of loudnesses in sones. The monotonic transformation perhaps succeeded in partialing out sensory values from (nonlinear) judgmental biases that operate in magnitude estimation.

Overall, the data agree reasonably well (though by no means fully) with the hypothesis that the dichotic summation of narrowly spaced tones involves a process of linear addition of loudnesses. If, however, loudness is counted in sones, then not only do the components add linearly, but loudness summation is complete.

\section{EXPERIMENT 2: LOUDNESS SUMMATION OF BINAURALLY PRESENTED NARROWLY SEPARATED TWO-TONE COMPLEXES}

The results of Experiment 1 suggest that over a relatively narrow frequency separation of components presented to the left and right ears, the rule of loudness summation remains roughly unchanged from the form it takes when no frequency separation is present (diotic or binaural summation). The narrow, subcritical-band fre- 
quency separation of the tones did not affect summation, presumably because the tones were presented dichotically, one to each ear. However, presenting the same tones diotically to both ears may change the rule of (linear) summation. Under such stimulus conditions, the largely peripheral critical-band mechanism is expected to impose limits on loudness summation. Hence, one does not expect linear loudness addition to prevail. For subcritical tones, like the ones used in Experiment 1, the expected rule is one of energy summation (rather than loudness summation). This prediction was tested in Experiment 2.

\section{Method}

Stimuli were produced as in Experiment 1, with one addition: The outputs of the oscillators combined at a mixer (Shure mixeramplifier unit) to produce the $1000+1100 \mathrm{~Hz}$ complex. The twotone complexes were presented binaurally (diotically) to both ears.

Again, the stimuli were constructed within the framework of a full, symmetrical factorial design. Each of six intensity levels of the $1000-\mathrm{Hz}$ component (including zero intensity) was combined with each of the same six intensity levels of the $1100-\mathrm{Hz}$ component, making 36 different stimuli in all. In a given session, the subject judged the entire matrix of 36 stimuli a total of four times. The stimuli were presented in an irregular order.

The procedure again consisted of free magnitude estimation. A total of 10 young men and women (5 of each, between 21 and 33 years of age) participated; 3 had served in Experiment 1 and another 2 had experience with the method of magnitude estimation.

\section{Results and Discussion}

Pooled results appear in Figure 4. The magnitude estimates assigned to each stimulus were brought to a common modulus and then averaged geometrically. These

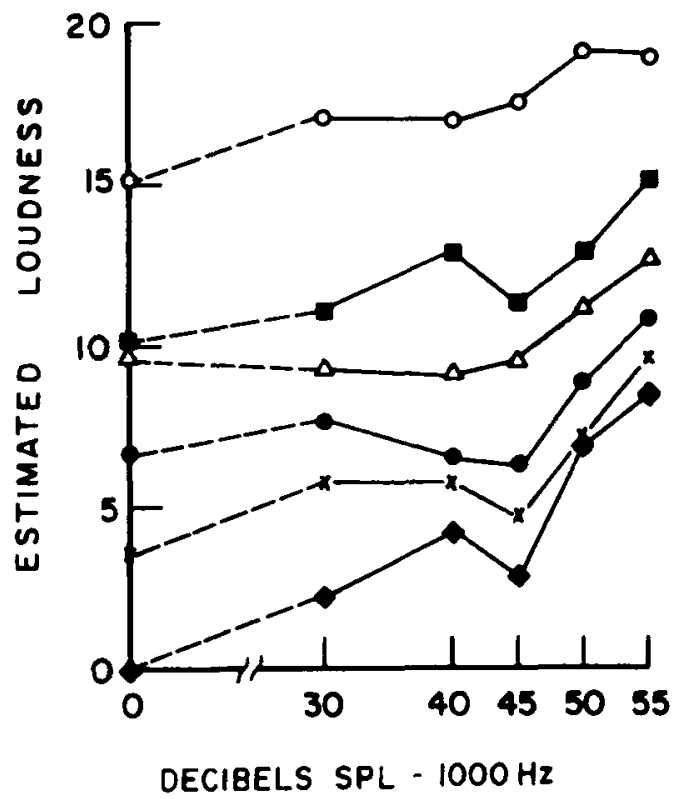

Figure 4. Magnitude estimates of the loudness of two-component sounds, presented binaurally to both ears. The estimates are plotted as a function of the six levels of the $1000-\mathrm{Hz}$ component, each of which was combined factorially with the same six levels of the 1100-Hz component. (From bottom in ascending order: 0, 30, 40, 45,50 , and $55 \mathrm{~dB}$ SPL.) means are plotted on a linear axis against the SPL of the $1000-\mathrm{Hz}$ component. Each contour represents a fixed SPL of the $1100-\mathrm{Hz}$ component. According to the rule of critical-band summation, loudness should depend solely on the total energy contained in each stimulus. That is, acoustical power components first add linearly, after which their sum undergoes a nonlinear transformation (by means of a negatively accelerated function) to produce the internal scale values directly proportional to loudnesses (e.g., Marks, 1979a, 1979b; Zwicker et al., 1957; Zwicker \& Scharf, 1965). Given that judgments of loudness are plotted on the linearly spaced ordinate of Figure 4, and that a single nonlinear transformation is applied to total acoustic power (i.e., the quantities that add are not loudnesses), the overall loudness estimates should not display additivity. That they do not is evident in the convergence at the upper-right portion of Figure 4. Adding a low level of one stimulus component to a low level of the other augments loudness notably, but adding a low level to a high one has virtually no effect on loudness. However, even though adding two approximately equalintensity components increases loudness, this does not double it. Analysis of variance confirms the lack of additivity: The overall interaction is significant $[F(25,225)=$ $2.02, p<.01]$. Thus the present estimates of narrowly spaced two-tone complexes result in an inherently nonadditive set of loudness judgments.

Marks (1979b) reported similar results for sinusoids of 1000 and $1090 \mathrm{~Hz}$, which were combined at different SPLs according to a factorial design of the type used here. He too found that the loudness of two-component tones lying close in frequency depends on the total energy. Since it is sound energy that sums linearly, loudness (the outcome of a nonlinear transformation applied to total energy) should not, perforce, yield a picture of linear additivity. The present set of data clearly demonstrates that it does not. The already well-known property of energy summation within a critical band (e.g., see Scharf,1970; Zwicker \& Scharf, 1965) has, therefore, gained support here.

\section{EXPERIMENT 3: MONAURAL SUMMATION OF NARROWLY SEPARATED TWO-TONE COMPLEXES}

Critical-band summation seems to represent peripheral processing that occurs at a stage prior to loudness summation. Presentation of a different frequency component to each ear (dichotic summation, Experiment 1) presumably bypasses this mechanism, enabling the system to display perfect additivity even for tones that fall close together in frequency. Presentation of both tones binaurally (diotic summation, Experiment 2), however, subjects them to an initial, critical-band-governed process, resulting in a nonlinear form of loudness integration. This suggests that delivering the narrowly spaced two-tone complexes to just one ear should yield results similar to those obtained for diotic summation-differing from those obtained for dichotic summation. Experiment 3, then, was 
an attempt to determine whether a monaurally presented, narrowly spaced two-tone complex, like a binaurally presented pair, displays imperfect summation in loudness units.

\section{Method}

The stimuli were produced as in Experiment 2, with one exception. The channel to the left ear was disconnected for all subjects. Thus, the $1000+1100 \mathrm{~Hz}$ complexes fed only the right ear.

The set of stimuli again consisted of the same factorial combination of intensities used in Experiments 1 and 2, delivered to the right ear. Each of 10 subjects, 4 men and 6 women between 17 and 30 years of age ( 4 of whom had served in at least one of the previous experiments) judged the entire matrix of 36 stimuli a total of four times each within the test session.

\section{Results and Discussion}

Figure 5 shows the pooled, normalized results. Considered overall, the present results are essentially the same as those found in Experiment 2, with the same tones presented binaurally. Again, distinct convergence at the upper right is evident. The departure from parallelism is reliable; the interaction term in the appropriate ANOVA is significant $[F(25,225)=2.14, p<.01]$. Taken as whole, the present data deviate notably from a rule of simple loudness addition. Of course, this result is the expected one, given the rule of energy summation within a critical band.

The results of Experiment 3 largely duplicate those of Experiment 2. They show that for components lying within a critical band the loudness is proportional to total acoustical power, shaped by means of a negatively accelerated function. This rule of energy summation within a critical band remains the same, regardless of whether the two-tone complex is presented to a single ear (as in

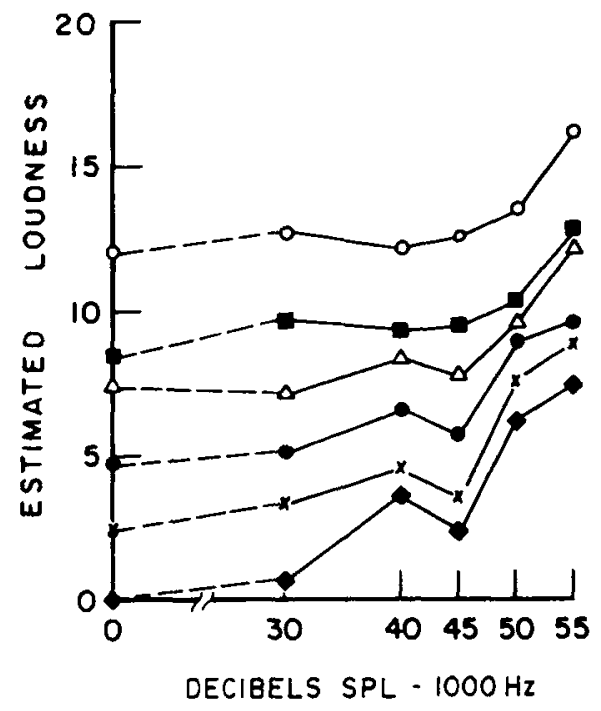

Figure 5. Monaural summation of the loudness of two-tone complexes $(1000-1100 \mathrm{~Hz}$ ). Magnitude estimates of the two-component tones, plotted against the SPL of the $1000-\mathrm{Hz}$ component. Each function represents a different SPL of the 1100-Hz component.
Experiment 3) or diotically to both ears (Experiment 2). It stands in sharp contrast, however, to the rule that governs dichotic summation, where a different frequency component is delivered to each ear (Experiment 1). There loudnesses summate between the two ears even when components are close together in frequency. It would appear, then, that the critical-band mechanism only affects monaural or diotic inputs. If this is true, the presentation of widely separated sound frequencies should bypass critical-bandwidth-bound processes even for monaural stimuli. Hence, for widely spaced complexes, both rules of summation and psychophysical contingencies should appear invariant across dichotic, diotic, and monaural modes of presentation. The following three experiments, patterned after the first three described above, served to test this prediction.

\section{EXPERIMENT 4: DICHOTIC LOUDNESS SUMMATION ACROSS WIDELY SPACED TONE FREQUENCIES}

Experiment 4 was an analogue, using widely spaced supercritical pure tones, to Experiment 1 on dichotic summation. Given the results of Experiment 1, which suggested that the complex with a narrow subcritical spacing showed virtually perfect summation, it was expected that a two-tone complex with a very wide frequency separation would give equally complete summation. On the other hand, there are indications in the literature (e.g. Zwicker et al., 1957; Zwislocki, Ketkar, Cannon, \& Nodar, 1974) that for wide spacing (especially at low levels), "loudness integration" may break down (but see Marks, 1979a, 1979b; Scharf, 1969). ${ }^{2}$ A more thorough empirical demonstration of the rule of across-frequency dichotic summation was, therefore, given in Experiment 4, which again consisted of a complete factorial design of intensities of the two components.

\section{Method}

The stimuli were produced as in Experiment 1 . We replaced the narrowly spaced tone pair with widely spaced, 2000 - and $5000-\mathrm{Hz}$ pure-tone stimuli. These components were presented dichotically, a different frequency to each ear (counterbalanced in a random fashion across ears and subjects). The stimuli consisted of the same factorial combination of six levels at $2000 \mathrm{~Hz}$ to one ear and six levels at $5000 \mathrm{~Hz}$ to the other ear as was used in Experiment 1.

Again, 10 young men and women participated ( 3 and 7 , between 17 and 32 years of age); 5 had served in at least one of the previous experiments. Each subject judged the entire matrix of 36 stimuli a total of four times within a test session, making 144 judgments in all.

\section{Results and Discussion}

Figure 6 shows the main results. The magnitude estimates were averaged geometrically, and the averages are plotted on a linear axis in Figure 6 as a function of SPL at $2000 \mathrm{~Hz}$ delivered to a single ear. Each contour represents a constant level of the $5000-\mathrm{Hz}$ component delivered to the other ear. The functions are parallel to one 


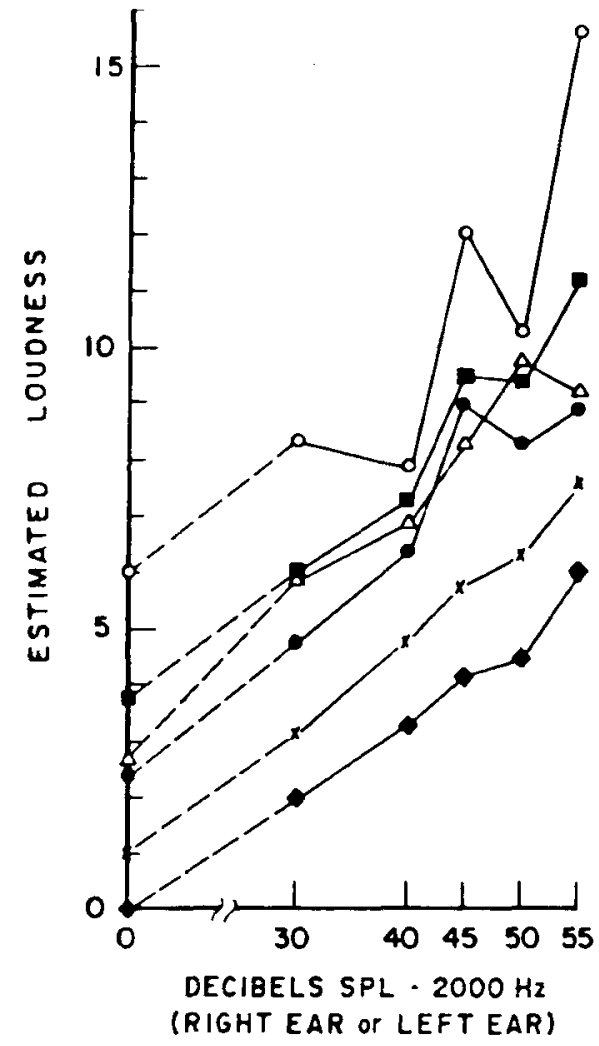

Figure 6. Dichotic summation of loudness. The factorial plot presents the data for the $\mathbf{2 0 0 0}+\mathbf{5 0 0 0 ~} \mathrm{Hz}$ two-tone complex. (From bottom in accending order: $0,30,40,45,50$, and $55 \mathrm{~dB}$ SPL.)

another (though a slight trend toward divergence at the upper right is evident), as they should be if they reflect linear addition. Analysis of variance yielded an overall interaction term that was nonsignificant $[F(25,225)=$ $1.28, p>.05]$. To a first approximation, then, these results imply that the subjects did resort to a simple addition process when they estimated total loudness.

Additivity can be seen even more clearly in the subset of data presented in Figure 7. Results for simple dichotic stimuli (triangles), and for the 2000- and $5000-\mathrm{Hz}$ monaural tones (open circles and $\times s$, respectively) closely follow the prediction of an additive model (filled circles). Although the monaural single-component functions for 2000 and $5000 \mathrm{~Hz}$ differ somewhat in this data set, judgments of their paired dichotic presentations equaled the sum of the judgments of the individually presented components nonetheless.

The data shown in the logarithmic coordinates of Figure 7 can be described fairly well by straight linesthat is, by power functions. The exponent of the $2000-\mathrm{Hz}$ (monaural) loudness function was $0.48\left(r^{2}=.918\right)$, whereas the loudness of the $5000-\mathrm{Hz}$ component increased as the 0.34 power of pressure $\left(r^{2}=.979\right)$. The simple dichotic function (equal SPL to the two ears) had an exponent of $0.50\left(r^{2}=.974\right)$. A more reliable indicant of the underlying loudness scales comes from taking the respective marginal means from the entire matrix of responses. The exponents have values of $0.52\left(r^{2}=.969\right)$ and $0.38\left(r^{2}=.962\right)$ for the $2000-$ and $5000-\mathrm{Hz}$ components, respectively. Yet a single power function with an exponent of $0.44\left(r^{2}=.974\right)$ is sufficient to describe both sets of data.

Dichotic summation can be assessed by averaging the separation in decibels between single-component and dichotic values over the $30-55 \mathrm{~dB}$ range depicted in Figure 7. The horizontal separation between the monaural and dichotic functions is about $12.5 \mathrm{~dB}$. That is, the monaural stimulus had to be about $12.5 \mathrm{~dB}$ greater than the dichotic stimulus to appear equally loud. This dichotic gain is significantly greater than $10 \mathrm{~dB}$-the gain expected for complete loudness summation (i.e., for a doubling of loudness)-if loudness is counted in sones. Recall that for the narrowly spaced complex in Experiment 1, dichotic gain averaged at around $10 \mathrm{~dB}$.

A particularly relevant experiment was conducted by Marks (1979a) on "cpgnitive summation." Left-ear and right-ear components in that study were separated by a long (2-sec) temporal interval, so the subject heard two distinct sounds, one to each ear. The subject had to integrate the two sensations consciously in order to judge their total loudness. Summation, then, was optional, not automatic, for it was induced by directing the subjects to add loudnesses. Interestingly enough, although the components added linearly, the average "binaural" gain was 12.5-the value obtained here for simultaneous dichotic presentation of widely separated components.

Zwicker et al. (1957) had already noted the difficulty of adding the loudnesses of widely spaced tones, saying that "it was apparently impossible for at least some sub-

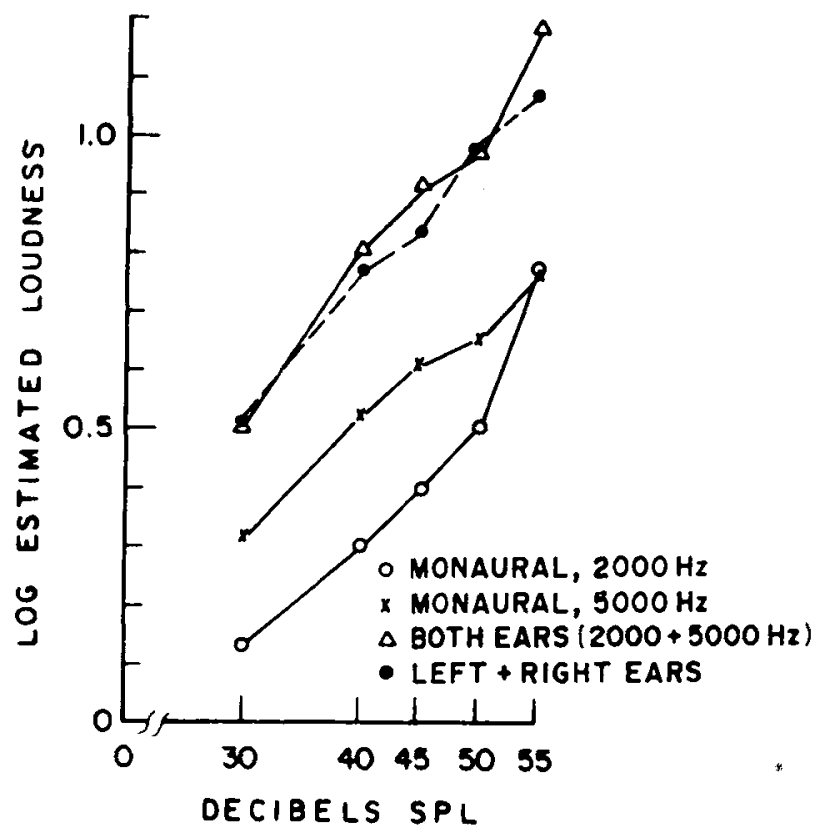

Figure 7. Magnitude estimates of the loudness of dichotic (equal SPL) and single-component monaural (2000 and $5000 \mathrm{~Hz}$ ) tonal stimuli. These data are subsets of the data in Figure 6. 
jects to 'integrate' the loudnesses of ... two disparate sounds" (p. 550). Scharf (1969) found that, over large frequency separations of components, most subjects hear two separate, localizable sound images rather than a single fused image. The splitting up of the dichotic complex alters the nature of the task considerably (see Békésy, 1960; Scharf, 1969). Summation under these circumstances is discretionary, coming under cognitive control. Thus, dichotic summation of widely separated components may represent yet another instance of what Marks (1979a) called cognitive summation (see also Algom \& CohenRaz, 1987). The results of the two studies fit together nicely in demonstrating linearly additive structures that operate on values of loudness that are clearly different from the sone scale.

The present results agree with those of Scharf (1969) in showing an additive model to govern dichotic summation of tones regardless of frequency spacing (compare the results of Experiment 1 with those of Experiment 3). Yet the present results hint that subjects may use a different scale of loudness when the dichotic complex is split up at the larger frequency separations. (Unfortunately, the use of loudness matching as the response did not permit Scharf to derive the appropriate loudness scale[s] in his study.) Less good agreement is found with Scharf's measurements on the amount of dichotic summation, which show a smaller gain-only 6-8 dB. Porsolt and Irwin (1967) and Niese (1960) similarly reported smaller degrees of summation, incompatible with the 10- to 12$\mathrm{dB}$ gains found here. An obvious difference between these earlier studies and our present one is in the makeup of stimuli. Only equal-intensity stimuli were employed in the previous studies, whereas a full matrix of equal and unequal levels to the two ears was used in Experiment 4. Beyond that methodological difference, however, the reason for the discrepancy is unclear.

\section{EXPERIMENT 5: DIOTIC SUMMATION OF WIDELY SEPARATED TWO-TONE COMPLEXES}

In Experiment 5, we tested the rule of summation for the widely spaced complex used in Experiment 4, presented diotically to both ears. Would estimates of total loudness still reveal the additive structure underlying the dichotic summation? Mutual interference, resulting in the rule of energy summation, does not affect widely spaced tones beyond the critical band (Scharf, 1969; Zwicker \& Scharf, 1965). Given the large frequency separation, the tones should, therefore, contribute fully to overall loudness despite their concurrent stimulation of a common cochlea.

\section{Method}

Sounds were generated in a manner identical to that of Experiment 2, except for the replacement of the narrowly spaced complex by the widely spaced $2000+5000 \mathrm{~Hz}$ complex. The stimuli constituted the same $6 \times 6$ factorial combination of SPLs used in
Experiment 2. Ten young men and women (4 and 6, between 18 and 30 years of age), 6 of whom had served in at least one of the previous experiments, participated. Again, all the stimuli were judged four times in the course of an experimental session.

\section{Results and Discussion}

Figure 8 gives the factorial plot. Despite few deviations, the functions in this family are nearly parallel. To a good first approximation, then, this set of data displays an additive structure. Indeed, the appropriate analysis of variance yields a nonsignificant interaction $[F(25,225)=$ $1.22, p>.05]$. Taken as a whole, the loudnesses of two sounds very far apart in frequency add linearly, so that their overall loudness equals the sum of the individual components.

In Figure 9, a subset of these data is plotted-namely, the results obtained when the $2000-$ and $5000-\mathrm{Hz}$ components were presented singly and in combination at equal levels. Again, the results agree virtually perfectly with the prediction of an additive model.

The curves in Figure 9 approximate power functions with nearly identical slope parameters. The exponents for the $2000-\mathrm{Hz}$, the $5000-\mathrm{Hz}$, and the simple binaural functions are $0.38\left(r^{2}=.925\right), 0.40\left(r^{2}=.910\right)$, and 0.40 $\left(r^{2}=.982\right)$, respectively. The exponents derived from

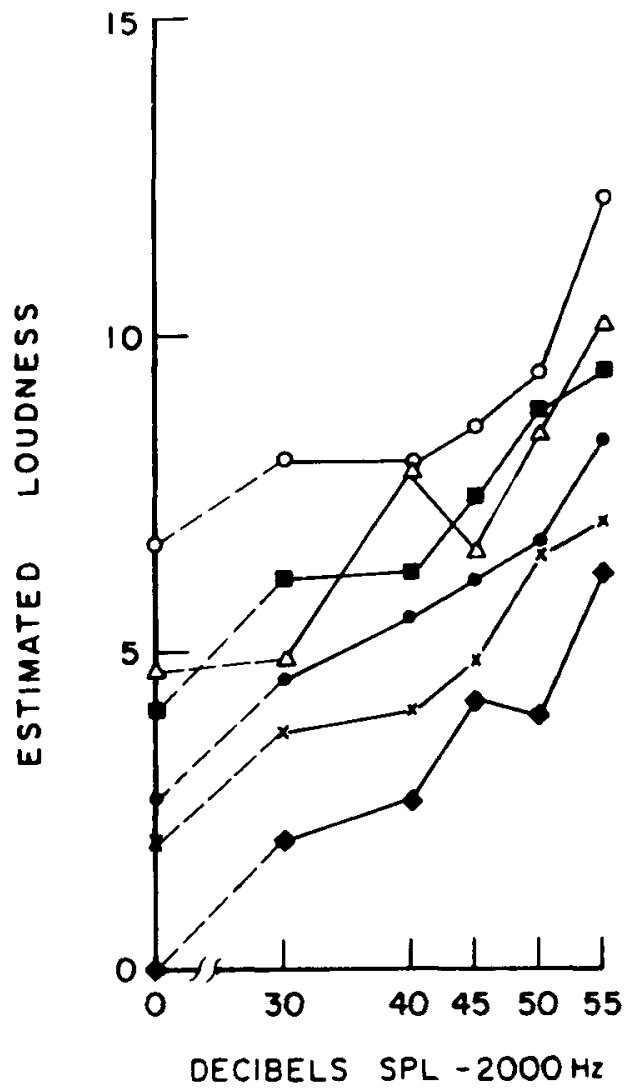

Figure 8. Magnitude estimates of diotic, two-component sounds. The factorial plot presents the data for a $2000+5000 \mathrm{~Hz}$ complex. (From bottom in ascending order: 0, 30, 40, 45, 50, and 55 dB SPL.) 


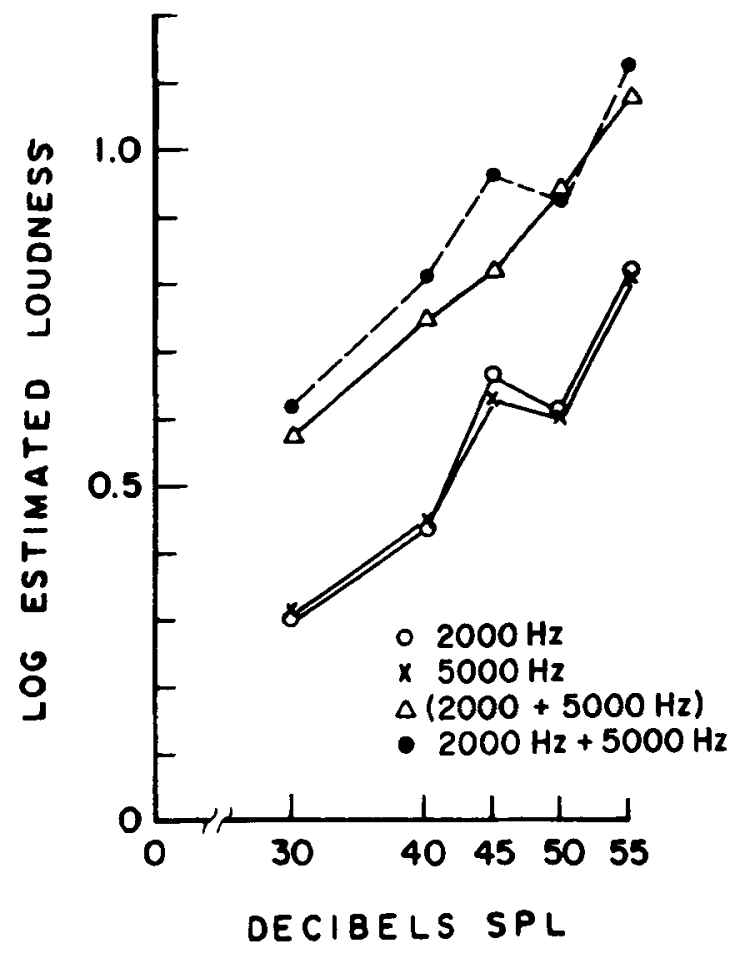

Figure 9. Magnitude estimates of one-component (2000 and $5000 \mathrm{~Hz})$ and two-component $(2000+5000 \mathrm{~Hz})$ sounds. These are subsets of the data in Figure 8.

the marginal means give similar values: $0.43\left(r^{2}=.987\right)$ and $0.44\left(r^{2}=.959\right)$, respectively, for the 2000 - and $5000-\mathrm{Hz}$ components.

The average horizontal separation between the singletone and the two-tone curves in Figure 9 is constant at around $10 \mathrm{~dB}$. This means that adding together the sounds of two equally loud tones that are far apart in frequency increases overall loudness to the same extent as does augmenting the SPL of either tone alone by $10 \mathrm{~dB}$. Loudness in sones also doubles with a 10- $\mathrm{dB}$ increase in sound pressure (Stevens, 1956). Therefore, not only is summation additive across widely spaced tones, it is also perfect in sones. ${ }^{3}$

Experiment 5 roughly replicated the findings of two earlier experiments by Marks (1978b, 1979b). In those studies, the components of widely spaced two-tone complexes of either 300 and $1000 \mathrm{~Hz}$ or 2000 and $5000 \mathrm{~Hz}$ took on several intensity levels, combined within a factorial design. Both studies gave nearly identical results: The loudness of the total equaled the sum of the perceived components. Moreover, both studies yielded psychophysi$\mathrm{cal}$ loudness functions with exponents at around 0.45 (except for the $300-\mathrm{Hz}$ stimulus)-compatible with the values derived here.

The results of Experiment 5 demonstrate that the loudness of a two-tone complex equals the sum of the component loudnesses, as they are heard in the complex (cf. Marks, 1979b, Scharf, 1969). Indeed, on the average, the ratio of the loudness of a simple complex (equal SPLs at the two frequencies) to the loudness of either component presented singly was $1.90: 1$. This contrasts sharply with the mutual masking that takes place when both components fall within a critical band, resulting in nonlinear subadditivity (Experiment 2). The next and final experiment duplicated the conditions of Experiment 5 with one exception: The values of the $2000+5000 \mathrm{~Hz}$ complex fed a single ear.

\section{EXPERIMENT 6: MONAURAL SUMMATION OF WIDELY SEPARATED TWO-TONE COMPLEXES}

\section{Method}

In Experiment 6, we used the widely spaced $2000+5000 \mathrm{~Hz}$ complex for compound, factorially combined, monaural (right-ear) presentations. Otherwise, Experiment 6 was identical to Experiment 3. Again, 10 young subjects ( 6 men and 4 women, between 18 and 32 years old) participated.

\section{Results and Discussion}

The mean judgments are shown in Figure 10. Despite some nonsystematic deviations, the six curves are essentially parallel, which means that these data are an additive function of the two supercritical frequency compo-

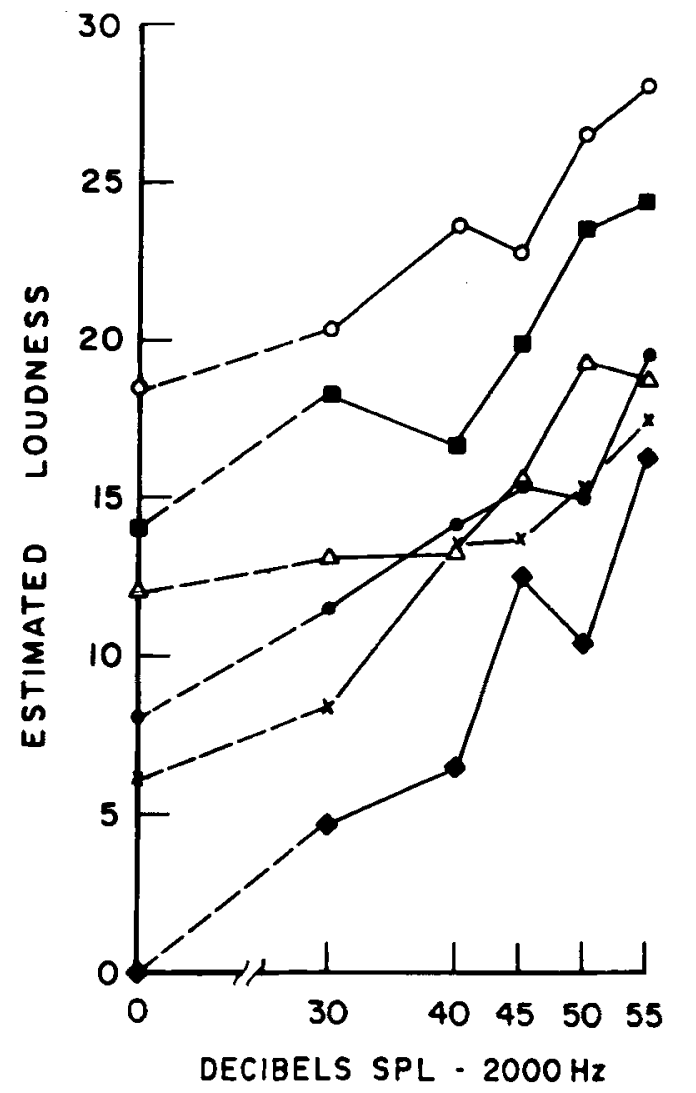

Figure 10. Monaural summation of the loudness of two-tone complexes. Factorial plot presents the data for the $2000+5000 \mathrm{~Hz}$ complex. (From bottom in ascending order: 0, 30, 40, 45, 50, and 55 dB SPL.) 


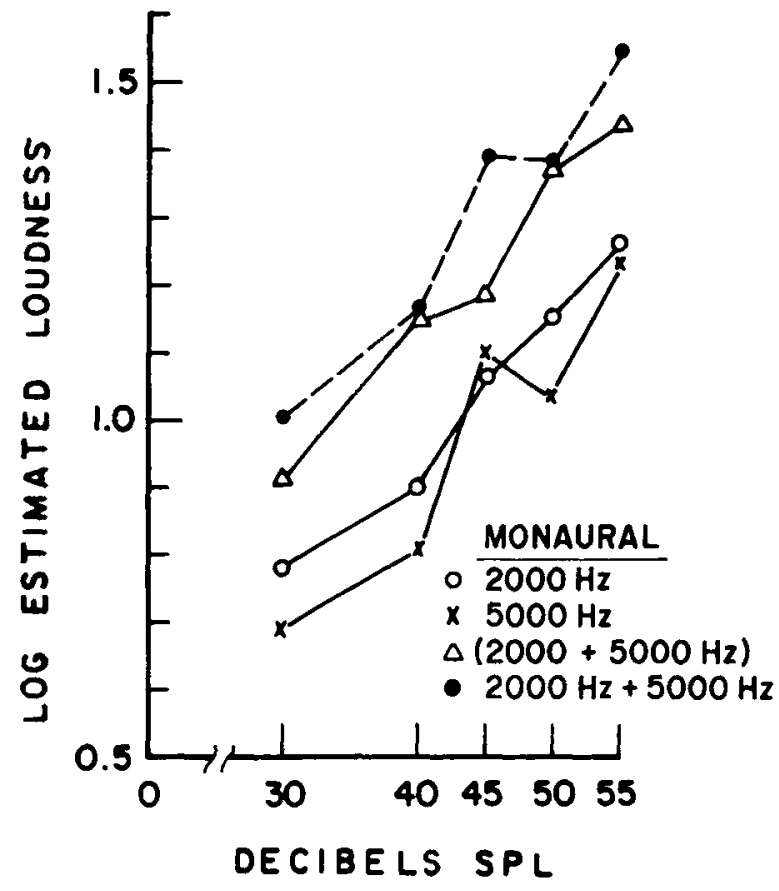

Figure 11. Magnitude estimates of the loudness of two-component $(2000+5000 \mathrm{~Hz})$ and one-component monaural $(2000$ and $5000 \mathrm{~Hz})$ stimuli.

nents. The $2000 \mathrm{~Hz} \times 5000 \mathrm{~Hz}$ interaction was significant at the $5 \%$ level $[F(25,225)=1.68, .01<p<.05]$, which confirms, though not on as strict a statistical level as before, the visual appearance of parallelism. Hence monaural presentation had no effect on the present widely spaced complex.

Figure 11 gives the loudness functions for the 2000$\mathrm{Hz}$, the $5000-\mathrm{Hz}$, and their simple (equal-SPL) combined presentations. All curves approximate power functions in the double-logarithmic coordinates. The respective exponents are $0.44,0.39$, and 0.44 . A single loudness function can fit both single-component data sets for values derived on the basis of the marginal means. This function has an exponent of 0.47 [the component exponents are $0.48\left(r^{2}=.955\right)$ for $2000 \mathrm{~Hz}$ and $0.46\left(r^{2}=.952\right)$ for $5000 \mathrm{~Hz}$ ]. It would seem, therefore, that a power function exponent of about 0.45 represents the growth of monaural loudness for all stimuli.

The average horizontal separation between single-tone and two-tone presentations averages at approximately $10 \mathrm{~dB}$. On the average, the subjects judged the loudness of the two-tone complex as 1.94 times greater than the loudness of either one of its equal-intensity components. The evidence for monaural additivity, acting on tones widely separated in frequency, seems strong. Thus, inherently different patterns of monaural loudness integration apply to subcritical tones (Experiment 3 ) as opposed to supercritical tones (Experiment 6). Only the latter display visibly additive metrics in which both components contribute their full share to overall loudness.
In a recent ingenious study, Schneider (1988) has also concluded that summation of loudness across critical bands is linear. His experiment bears some similarity to the present Experiment 6, in that Schneider had subjects compare the loudnesses of various combinations of 2- and 5$\mathrm{kHz}$ complexes. He used a nonmetric paired-comparison technique, in which subjects judged which of two 2-and $5-\mathrm{kHz}$ combinations sounded louder. The matrix of results, analyzed under the rubric of the theory of conjoint measurement (e.g., see Krantz, Luce, Suppes, \& Tversky, 1971), was consistent with the hypothesis that the auditory system sums the sensory effects of concurrent supercritical stimulation linearly. Schneider derived loudness scales from the paired-comparison data that could be described as power functions of sound pressure; the average exponent equaled about 0.6 . Due to the method used and the lack of an error theory, however, Schneider could neither test the observed violations of additivity nor specify an average value of the gain in decibels between equally loud single-frequency (2-or 5-kHz) and complex (2- and $5-\mathrm{kHz})$ tones. Both are gauged in our Experiment 6 .

\section{GENERAL DISCUSSION}

The objectives of this study were relatively modest. We conducted a systematic investigation of various loudnesssummation phenomena within the framework of a single experimental paradigm. Earlier studies of the auditory integration processes examined here had been carried out in different experiments, indeed in different laboratories. Most importantly, we applied a procedure different from that used in most previous measurements of loudness summation. We used magnitude estimates in conjunction with a multivariate model of sensory integration, in order to derive rules of composition and the associated scales of loudness. Indeed, the joint derivation of (1) measures of summation and (2) loudness scales is the signature of the present methodology. Specification of the former sufficiently constrains the latter, conferring validational support on the overt numerical estimates (e.g., Algom, Palmon, \& Cohen-Raz, 1989; Algom, Rubin, \& CohenRaz, 1989). The logic behind the present approach owes much to Anderson's (1970) functional measurement theory, but it is also compatible with other multivariate models of sensory processes such as conjoint measurement (Krantz et al., 1971).

The present results support the notion of a critical-band mechanism conceived as a peripheral filter operating on the monaural input (cf. Scharf, 1961, 1970). Whether two narrowly spaced tones obey the intensity-summation principle (loudness is a function of total sound intensity; Experiments 2 and 3 ) or the loudness-summation principle (loudness equals the sum of the component loudnesses; Experiment 1) therefore depends on whether or not they are stimulating the same cochlea. By the same token, for wide spacing of frequency, loudness summation applies 
regardless of mode of presentation (dichotic, diotic, or monaural; Experiments 4-6). ${ }^{4}$ Examination of the data from Experiments 2 and 3-the only conditions for which intensity summation should hold-indeed shows significant deviations from parallelism predicted by the principle of loudness summation. Moreover, the pattern of data in these two conditions differs considerably from that obtained in the other experiments. In general, the present set of experiments allowed for six independent tests of the operative integration rule (additive vs. nonadditive). In all cases, the particular form that loudness summation assumed followed the prediction derived from the concept of a peripheral critical-band mechanism. Chance probability for this result is approximately $1.5 \%$. If we combine the results of the present experiments with two comparable conditions from Algom and Marks (1984) and Marks (1978a) on binaural summation, and with two additional experiments from Marks (1979b) on crossfrequency summation for sub- and supercritical tones, we have 10 independent comparisons, differing across subjects and stimulus selection. Yet all yield metric structures and psychophysical functions compatible with a model in which either a wide spacing of frequency components to a single ear or the splitting of narrowly spaced components to separate ears can bypass the constraints posed by a peripheral, bandwidth-bound, energy-driven mechanism. The chance probability for such an outcome is .00098 .

No measurement is free of errors and biases, and the present procedures are no exceptions. Thus, their advantages notwithstanding, magnitude estimates are also less precise than traditional methods such as matching or adjustment. For example, magnitude estimation cannot be expected to resolve differences among experiments of the order of single decibels. Even more disturbing is another feature that such data typically generate-namely, great interindividual variation. Unfortunately, in this respect, loudness estimates were quite variable in large portions of the present data. Moreover, systematic biases appeared as well. Thus, if the data in Experiments 2 and 3 are to be fully explained by intensity summation, then tone pairs with equal energy should have equal loudness. However, when we plotted (separately for each experiment) the loudness estimates against total energy, the points were not dispersed in a completely random fashion around the best fitting line. This result implies that strict intensity summation may not hold. For loudness summation too, there were occasionally systematic departures from complete additivity (see Figure 2), although the support for linear summation here is much stronger. Therefore, a more conservative summary of the present data taken as a whole would be that departures from additivity in Experiments 1 and 4-6, if any, are minor relative to the departures from additivity shown in Experiments 2 and 3 . These findings provide additional support for the notion that summation is different within and across critical bands.

\section{REFERENCES}

Algom, D., Adam, R., \& Cohen-R^z, L. (1988). Binaural summation and lateralization of transients: A combined analysis. Joumal of the Acoustical Society of America, 84, 1302-1315.

Algom, D., \& CoHen-RAz, L. (1984). Visual velocity input-output functions: The integration of distance and duration onto subjective velocity. Journal of Experimental Psychology: Human Perception \& Performance, 10, 486-501.

Algom, D., Cohen-Raz, L. (1987). Sensory and cognitive factors in the processing of visual velocity. Journal of Experimental Psychology: Human Perception \& Performance, 13, 3-13.

ALGOM, D., MARKs, L. E. (1984). Individual differences in loudness processing and loudness scales. Journal of Experimental Psychology: General, 113, 571-593.

Algom, D., Palmon, R., Cohen-Raz, L. (1989). Loudness scales from loudness processes: A multivariate approach. International Journal of Neuroscience, 46, 167-183.

Algom, D., Rubin, A., Cohen-RAz, L. (1989). Binaural and temporal integration of the loudness of tones and noises. Perception \& Psychophysics, 46, 155-156.

Anderson, N. H. (1970). Functional measurement and psychophysical judgment. Psychological Review, 787, 153-170.

ANDERSON, N. H. (1982). Methods of information integration theory. New York: Academic Press.

BÉKÉsY, G. VON. (1960). Experiments in hearing. New York: McGraw-Hill.

IRWIN, R. J. (1965). Binaural summation of thermal noises of equal and unequal power in each ear. American Journal of Psychology, 78, $57-65$.

Krantz, D. H., Luce, R. D., SupPes, P., \& Tversky, A. (1971). Foundations of measurements: Vol. 1. Additive and polynomial representations. New York: Academic Press.

Lane, H. L., Catania, A. C., \&tevens, S. S. (1961). Voice level: Autophonic scale, perceived loudness, and effect of side tone. Journal of the Acoustical Society of America, 33, 160-167.

MArKs, L. E. (1978a). Binaural summation of the loudness of pure tones. Journal of the Acoustical Society of America, 64, 107-113.

MArks, L. E. (1978b). Phonion: Loudness scales and properties of auditory intensity. In N. J. Castellan \& F. Restle (Eds.), Cognitive theory (Vol. 3, pp. 7-31). Hillsdale, NJ: Erlbaum.

MARKS, L. E. (1979a). Sensory and cognitive factors in judgments of loudness. Journal of Experimental Psychology: Human Perception \& Performance, 5, 426-443.

Marks, L. E. (1979b). A theory of loudness and loudness judgments. Psychological Review, 86, 256-285.

MARKS, L. E. (1980). Binaural summation of loudness: Noise and twotone complexes. Perception \& Psychophysics, 27, 489-498.

Marks, L. E. (1987). Binaural versus monaural loudness: Supersummation of tone partially masked by noise. Journal of the Acoustical Society of America, 81, 122-128.

NiEse, H. (1960). Subjektive Messung der Lautstärke von Bandpassrauschen. Hochfrequenztechniques und Elektroakustiks, 68, 202-217.

Porsolt, R. D., IRWN, R. J. (1967). Binaural summation in loudness of two tones as a frequency of their bandwidth. American Journal of Psychology, 80, 384-390.

Reynolds, C. S., Stevens, S. S. (1960). Binaural summation of loudness. Joumal of the Acoustical Society of America, 32, 1337-1344.

SCHARF, B. (1959). Critical bands and the loudness of complex sounds near threshold. Joumal of the Acoustical Society of America, 31, 365-370.

SCHARF, B. (1961). Complex sounds and critical bands. Psychological Bulletin, 58, 205-217.

SCHARF, B. (1968). Binaural loudness summation as a function of bandwidth. In Y. Kohasi (Ed.), Reports of the Sixth International Congress of Acoustics, Tokyo, A-3-5, pp. 25-28.

SCHARF, B. (1969). Dichotic summation of loudness. Journal of the Acoustical Society of America, 45, 1193-1205. 
SCharf, B. (1970). Critical bands. In J. V. Tobias (Ed.), Foundations of modern auditory theory (Vol. 1, pp. 159-202). New York: Academic Press.

SChARF, B. (1978). Loudness. In E. C. Carterette \& M. P. Friedman (Eds.), Handbook of perception (Vol. 4, pp. 187-242). New York: Academic Press.

SCHARF, B., FishKeN, D. (1970). Binaural summation of loudness: Reconsidered. Journal of Experimental Psychology, 86, 374-379.

SCHNEIDER, B. (1980). Individual loudness functions determined from direct comparisons of loudness intervals. Perception \& Psychophysics, 8, 493-503.

SCHNEIDER, B. (1988). The additivity of loudness across critical bands: A conjoint measurement approach. Perception \& Psychophysics, 43, 211-222.

STEVENS, S. S. (1956). The direct estimation of sensory magnitudeloudness. American Journal of Psychology, 69, 1-15.

ZWTCKer, E., FlotTorP, G., \& STEvens, S. S. (1957). Critical bandwidth in loudness summation. Journal of the Acoustical Society of America, 29, 548-557.

ZWICKER, E., SCHARF, B. (1965). A model of loudness summation. Psychological Review, 72, 3-26.

Zwislocki, J. J., Ketkar, I., Cannon, M. W., Nodar, R. H. (1974). Loudness enhancement and summation in pairs of short sound bursts. Perception \& Psychophysics, 16, 91-95.

\section{NOTES}

1. Beyond these rudimentary principles, however, many uncertainties mark virtually every aspect of the summation phenomena. For example, Marks (1978a, 1979a, 1979b, 1980) and Algom and Marks (1984) have reported complete binaural summation for tones, whereas Scharf (1969) and Scharf and Fishken (1970; see also Scharf, 1978) have only found partial summation. With respect to binaural summation of multitone complexes, Scharf (1968) reported increased summation in decibels with increased overall frequency separation of the components. Marks (1980), on the other hand, reported less summation for a widely spaced two-tone complex ( 300 and $4800 \mathrm{~Hz}$ ) than for a more narrowly spaced complex (675 and $1475 \mathrm{~Hz}$ ). For widely spaced monaural (or diotic) two-tone complexes, Zwislocki et al. (1974) and Marks (1979b) reported loudness summation equivalent to a gain of $10 \mathrm{~dB}$ (at around $1000 \mathrm{~Hz}$ ), which is a doubling of loudness on the sone scale. Scharf $(1970,1978)$ however, reported gains greater than 10 phons for widely separated tone complexes at intermediate intensity levels. For dichotic stimulation, Scharf (1969) reported less loudness summation at the center frequency of $500 \mathrm{~Hz}$ than at $2000 \mathrm{~Hz}$; Porsolt and Irwin (1967), however, found just the opposite. Finally, the dependence on level of both binaural and dichotic summation is still moot (compare the results reported by Porsolt and Irwin, 1967, and Scharf, 1969, with those reported by Marks, 1978a, 1979a, 1979b).

2. It should be pointed out that Zwicker et al. (1957) studied diotic summation. Nevertheless, their argument about the difficulty of integration when the tones are "faint and widely separated" is a general one, so that "it may be" that for low-level and wide spacings, "loudness integration, whatever that is, tends to break down" (p. 550). The outcome may differ, however, for monaural stimuli (see Scharf, 1959).

3. A point should be made here about the compatibility of the derived binaural gain (about $10 \mathrm{~dB}$ ) with the relatively small exponents (at around 0.42 ). Although the present data display the properties of an additive model, this model did not yield a tightly constrained metric (numeric) structure in the present case. This indeterminancy, resulting from certain noisiness in the data, may explain the appearance of smaller exponents that the 0.6 value prescribed for sones.

4. Formally, the loudness $L_{\mathrm{G}}$ of two tones with intensities $I_{1}$ and $I_{2}$ lying close together in frequency (i.e., within a critical band) is given by

$$
L_{\mathrm{G}}=f\left(I_{1}+I_{2}\right) \text {, }
$$

where $f$ is the psychophysical function transforming their total intensity into loudness. By contrast, the loudness of two tones widely separated in frequency (i.e., beyond a critical band) is given by

$$
L_{\mathrm{G}}=f\left(I_{1}\right)+f\left(I_{2}\right),
$$

where total loudness equals the sum of the loudnesses of the component tones. We thank an anonymous reviewer for suggesting this summary.

(Manuscript received December 12, 1988; revision accepted for publication June 21,1989 .) 